\title{
The effect of isokinetic and proprioception training on strength, movement and gait parameters after acute supination injury of the ankle ligaments
}

\begin{abstract}
The effects of a three-week isokinetic training compared to typical proprioceptive training on parameters of strength, movement and gait function after acute ankle ligament sprain were investigated.

C Mucha (PhD)

Thirty-nine patients were randomly allocated to two comparison groups. In group $1(n=20)$ a proprioceptive training and in group $2(n=19)$ an isokinetic strength training (Cybex 6000@) were administered. The patients of both groups underwent training five times a week for three weeks. Before and at the end of the treatment course, in both groups isokinetic strength was tested, the range of motion in the ankle joint was recorded and gait was analyzed (multicomponent strength measurement platform, Henschel-System $\left.{ }^{\circledR}\right)$. The maximum isokinetic torque $(60 \% \mathrm{~s})[\mathrm{Nm}]$ and the contact time (monopedal support time) of the injured leg during gait cycle were the basis for evaluation.

The data obtained show that in group 2 a significantly greater increase of the maximum isokinetic torque was attained in almost all range of motion of the ankle joint in the course of treatment. At the same time, in group 2 the shortening of the contact time in the stance phase of the injured leg could be compensated. The active range of motion in the ankle joint was less at the end of treatment in group 2 than in group 1. The isokinetic training obviously did not only lead to better strength regeneration, but also to a functionally more stable ankle joint with a rhythmically more evenly balanced stance phase of the gait cycle. These results suggest that the used isokinetic training had positive effects on functional stability after acute ankle sprain.
\end{abstract}

KEYWORDS: ANKLE JOINT, SUPINATION INJURY, REHABILITATION, PROPRIOCEPTIVE VERSUS ISOKINETIC TRAINING.

\section{INTRODUCTION}

Supination injuries of the ankle ligaments are common injuries as Berlemann et al (2000), refer and the most frequent injuries in sports. However, a ligament rupture (mostly to the anterior talofibular ligament) occurs in only $10 \%$ of all traumata. Good results under conservative treatment have been described by Mattacola and Dwyer (2002) for mild and moderately severe injuries on the lateral ligament apparatus of the ankle joint. Early mobilization that entails supporting measures such as taping, bandaging, orthotics and if necessary plaster cast is thought to promote healing after only a brief phase of immobilization. Early mobilization is intended above all to stimulate neuromuscular control of the foot, which is why proprioceptive training is strongly recommended in the exercise protocol of
Matsusaka et al (2001). However van der Wees et al (2006) demonstrated that these exercise programs are effective in the prevention of recurrent ankle sprains only. In a review of recent studies Zöch et al (2003) came to the result, that a combination of different exercises, including strengthening of the peroneal musculature, leads to better results and allows earlier return to the activities of daily life.

Strengthening of the peroneal musculature is also recommended by Uh et al (2000). Weinstein (1993) demonstrated that insufficient strength of the peroneal muscles is associated with chronic ankle instability and recurrent injury. Whereas numerous studies have been published on proprioceptive training, studies on the effect of specific muscle training can hardly be found in the literature. The need arises for studies that test the effects of an isokinetic training programme on discrete parameters of regeneration after acute supination injury of the ankle joint which will be compared with the more commonly used proprioceptive training programmes.

The aim is to investigate whether an isokinetic strength training leads to better effects on parameters of functional stability after acute ankle sprain than the commonly used proprioceptive training.

\section{Correspondence to:}

Univ.-Prof. Dr. med. C. Mucha Deutsche Sporthochschule Köln Abt. Medizinische Rehabilitation und Prävention

Am Sportpark Müngersdorf 6 50933

Köln

Email: Tech@dshs-koeln.de 


\section{Patients and Methods}

Thirty-nine patients with acute supination injury of the ankle ligaments have been examined in two selected surgical centers, where the diagnose fibular capsule ligament lesion grade II was made and functional treatment started. After four weeks these patients were randomly assigned to two different rehabilitation programs for further three weeks in our rehabilitation center.

Patient consent was secured in compliance with the institution's clinical research and investigations committee guidelines. The guidelines for the following rehabilitation programs were established with the treating therapists prior to initiating the study. In accordance with rehabilitation protocols of Chaiwanichsin et al (2005) in group 1 $(n=20)$ the exercise program focused on proprioception training (balance exercises, wobble board training, walking and running exercises on soft floor, mats, inclined plane with variations of pace. In group $2(n=19)$, isokinetic strength training was conducted. The Cybex $6000 \AA$ was used for isokinetic strength training; the exercise positions also corresponded to the test positions. Plantar flexion and dorsal extension exercises were from the prone position with extended leg. The inversion and eversion exercises were performed in the prone position with flexed hip and knee joints. Exercise and test rate were set at $60 \%$ s. The treatment took place in both groups in synchrony with working days five times a week. The time for each session takes 30 minutes. On admission, patients' demographic data and case history were taken, especially with regard to the injury and its treatment in the surgical centers.

At the beginning and at the end of the three-week controlled treatment course, the following were investigated: isokinetic strength test, gait analysis parameters and range of motion.

In the isokinetic strength test, the maximum isokinetic torque $(60 \%)$ s $[\mathrm{Nm}]$ was calculated, since it is regarded as a reliable parameter in isokinetic strength measurements like stated by Kaminski et al (2003). It enables the greatest strength development during one exercise cycle. The maximum torque also constitutes the basis for other strength parameters. In accordance with the recommendations on Lelie et al (1990) five movements were measured as a basis for calculating the arithmetic mean. The range of motion was registered according to the neutral 0 method in the ankle joint with the Cybex 6000®. Gait was analyzed using two multicomponent strength measurement platforms (Henschel System ${ }^{\circledR}$ ) set into the floor, which enabled a two-step analysis. Several parameters of the gait cycle registered indicated that the contact time (monopedal support time) was the most important parameter. Cochran (1988) demonstrated that it reacts particularly

Table 1: Range of motion in the ankle joint in the comparison groups at the final examination (T2)

\begin{tabular}{|l|l|l|}
\hline $\begin{array}{l}\text { Range of } \\
\text { motion }\end{array}$ & $\begin{array}{l}\text { Group 1 } \\
\overline{\boldsymbol{X}} \pm \mathbf{~ S ~}\left[{ }^{\circ}\right]\end{array}$ & $\begin{array}{l}\text { Group 2 } \\
\overline{\boldsymbol{X}} \pm \mathbf{~ S ~}\left[\angle^{\circ}\right]\end{array}$ \\
\hline Plantarflexion & $33,6 \pm 7,7$ & $31,2 \pm 5,8$ \\
\hline Dorsi extension & $26,7 \pm 5,2$ & $26,3 \pm 5,9$ \\
\hline Inversion & $42,0 \pm 7,7$ & $38,6 \pm 7,2$ \\
\hline Eversion & $38,3 \pm 6,3$ & $36,7 \pm 9,1$ \\
\hline
\end{tabular}

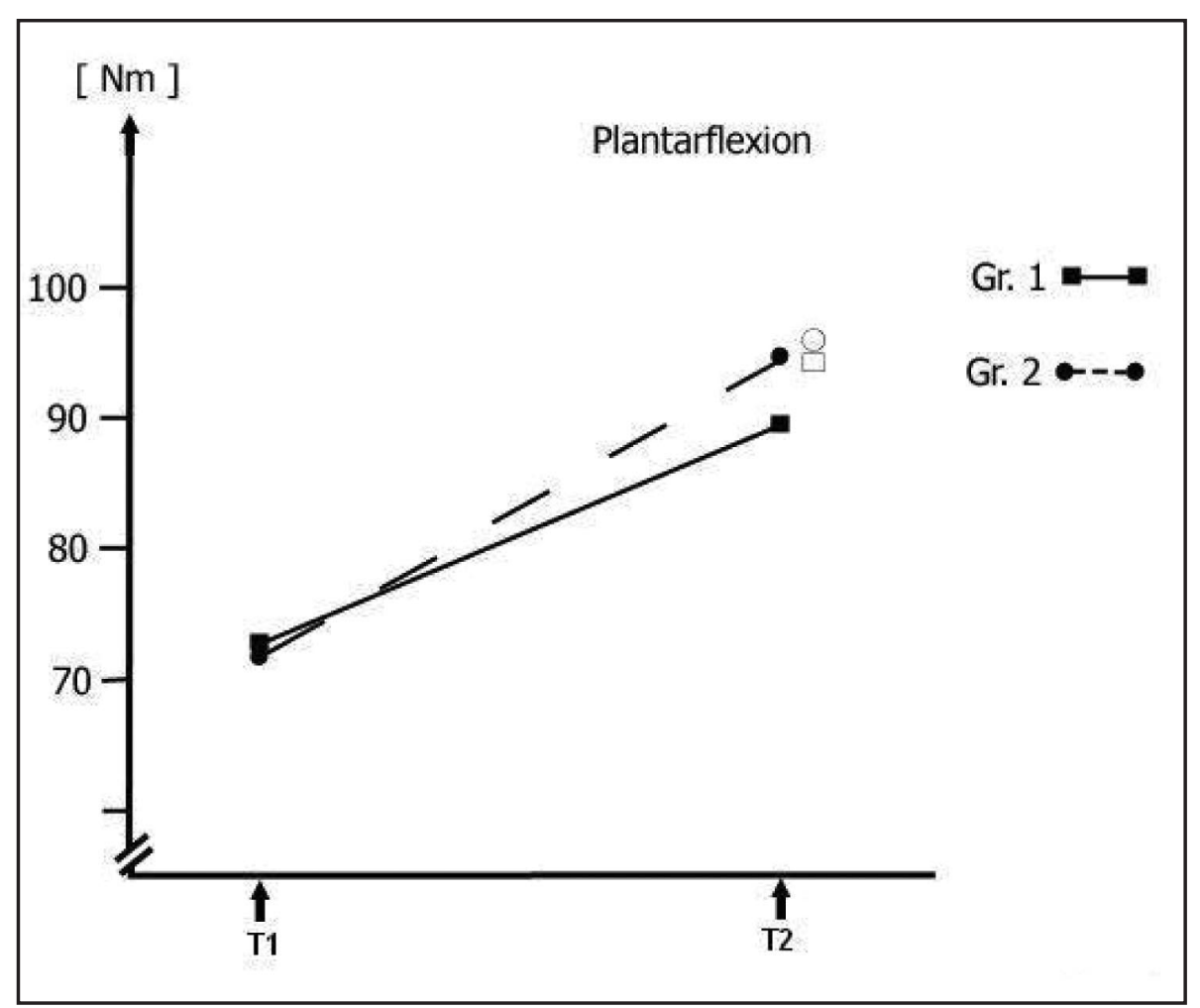

Figure 1: Maximum isokinetic torque in plantar flexion of the injured ankle in the comparison groups at the beginning of the investigation $\left(T_{1}\right)$ and at the end of the investigation $\left(T_{2}\right)(\square 0=$ maximum isokinetic torque on the uninjured side). 


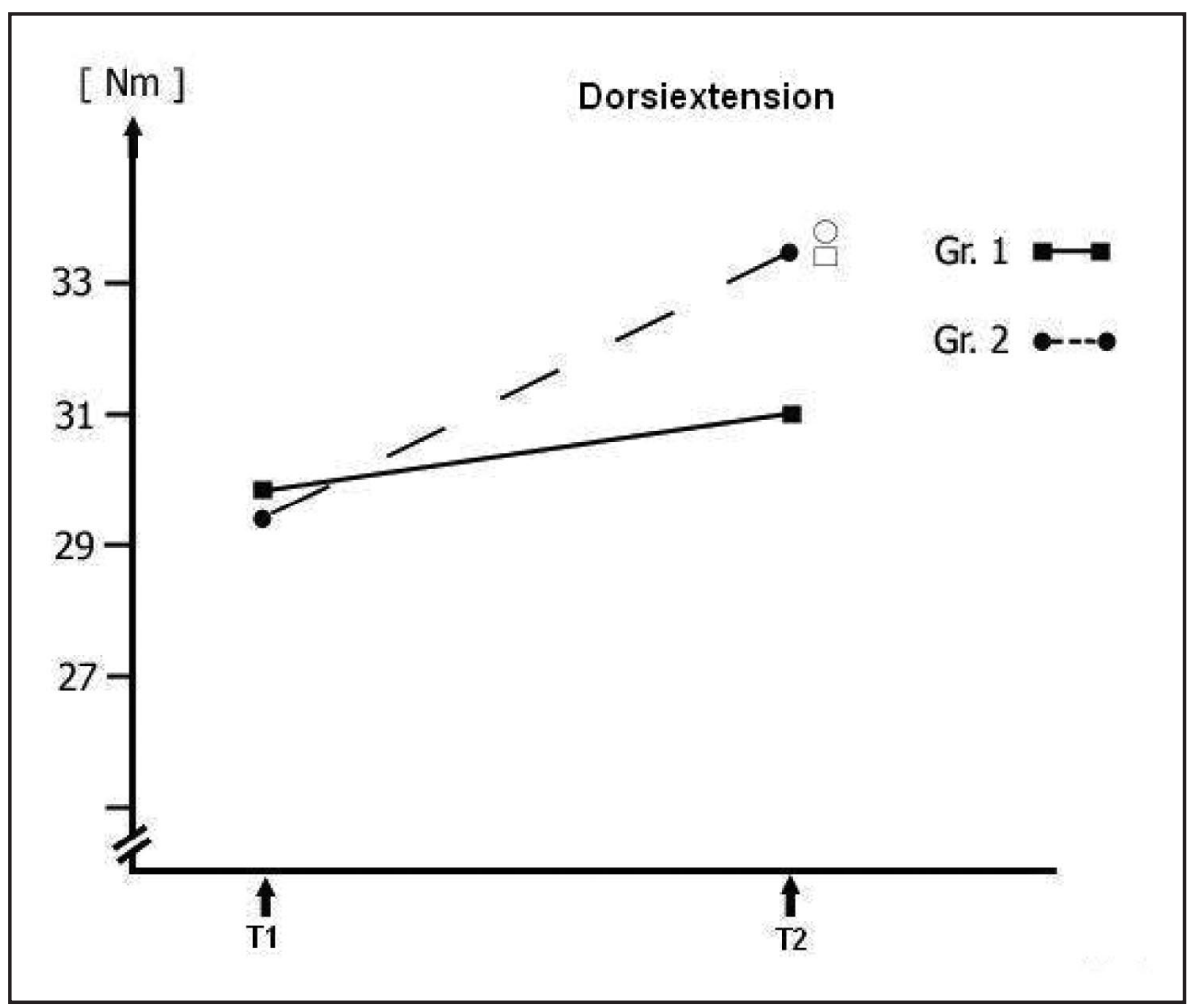

Figure 2: Maximum isokinetic torque in dorsi extension of the injured ankle in the comparison groups at the beginning of the investigation $\left(T_{1}\right)$ and at the end of the investigation $\left(T_{2}\right)$ ( $\square 0=$ maximum isokinetic torque on the uninjured side).

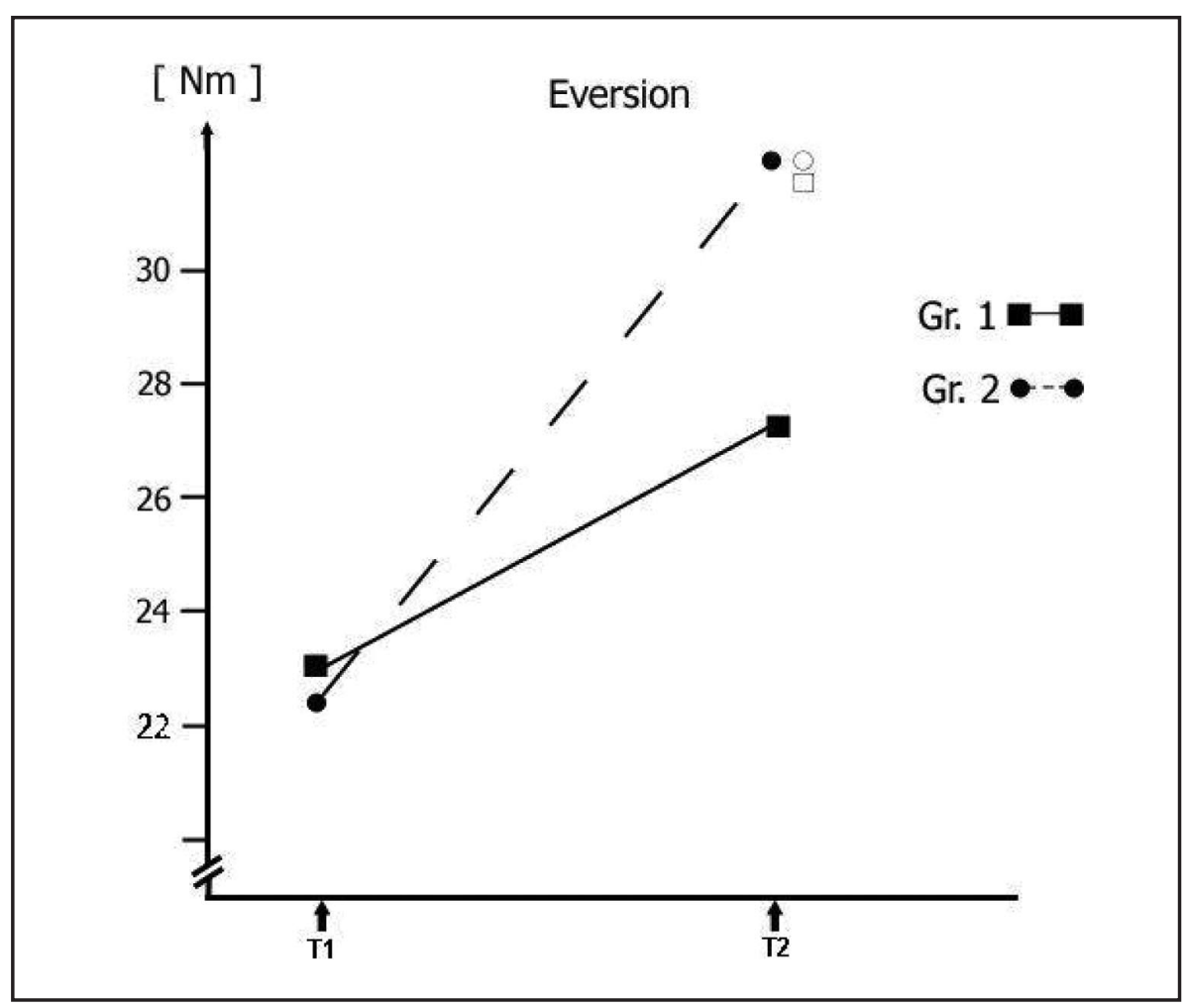

Figure 3: Maximum isokinetic torque in the eversion of the injured ankle in the comparison groups at the beginning of the investigation $\left(T_{1}\right)$ and at the end of the investigation $\left(T_{2}\right)(\square 0=$ maximum isokinetic torque on the uninjured side).

\section{RESULTS}

Thirty-nine patients (12 women and 27 men) with an average age of $25 \pm 7.2$ years were included in the investigation. The majority of these patients had suffered a sport accident (27\% football, $19 \%$ volleyball) and the remainder had everyday accidents or had engaged in various kinds of recreational sport. According to the data from the case history, all the patients had a fresh supination injury with a fibular capsule ligament lesion (grade II) that was given conservative functional treatment. Four weeks after the trauma, the patients were included in the study and assigned to the two reference groups: 20 patients to group 1 and 19 patients to group 2 . Since one patient of group 2 had to discontinue the control therapy for family reasons, the results of only 18 patients were evaluated in group 2 .

The maximum isokinetic torque for the individual motion amplitude at the injured ankle joint is shown in Figures 1 - 4 for both groups. Apart from that in inversion, a very much greater increase of the maximum isokinetic torque could be attained in group 2, which underwent isokinetic strength training, than in group 1, in which the typical proprioceptive training was continued. The difference was especially pronounced and statistically significant $(\mathrm{p}<0.05)$ in range of dorsi extension and in eversion. In the final examination (T2), group 2 achieved a maximum isokinetic torque on the injured side, which corresponded to that of the healthy side. In contrast, in group 1, it was still substantially below that of the healthy side. The increase of the maximum isokinetic torque in both groups was roughly the same only for the range of inversion.

Table 1 shows the range of motion in the ankle joint of the two groups. At the end of treatment, group 2 showed slightly smaller amplitudes in almost all range motions than group 1 . The most striking difference was in the extent of inversion.

Figure 5 shows the mean contact time on the injured side in the comparison groups at the time of the first (T1) and the second (T2) examination. The mean contact time could be prolonged after three weeks of therapy in both groups, 
but the prolongation was more pronounced in group 2, in which it was indeed almost the same as that on the healthy side. The prolongation of the contact time in group 2 was statistically significant $(\mathrm{p}<0.05)$.

\section{DISCUSSION}

With respect to their age and gender distribution and the cause of the injury, patients in this investigation largely corresponded to those reports in epidemiological studies of Lohrer et al (2000) and Rammelt et al (2003). Typically, the supination injury of the ankle joint mainly occurred in young patients as a result of a sport accident and was more frequent in men than in women.

Treatment in primary surgery departments with conservative functional treatment comprised the typical three phases: short immobilization in elevation of the leg, cooling and compression of the injured ankle and subsequent early mobilization with adaptations of weightbearing and with supporting taping, bandages or ortheses. Four weeks after the injury, the patients entered the study and were assigned to one of the two therapy groups. In the course of the study lasting three weeks, the treatments in the groups were carried out by the same therapists. In group 1, the exercise structure mainly comprised exercises for proprioception in accordance with the recommendations of rehabilitation protocols of Chaiwanichsin et al (2005). These were supplemented by balance, gait and coordination exercises as well as strength exercises for the peroneal musculature and performed in the form of circuit training. In group 2, isokinetic training on the Cybex $6000 ®$ took place instead. This was also carried out with $60 \%$ just like the diagnostic strength tests, since this training rate is generally considered especially suitable for the purposes of rehabilitation.

Besides the improvement of proprioception, the need to increase the strength of the lower-leg musculature that actively stabilizes the ankle joint, especially that of the peroneal muscles, is referred to by Berlemann et al (2000) and is also taken into consideration in the rehabilitation protocols of Zöch et al

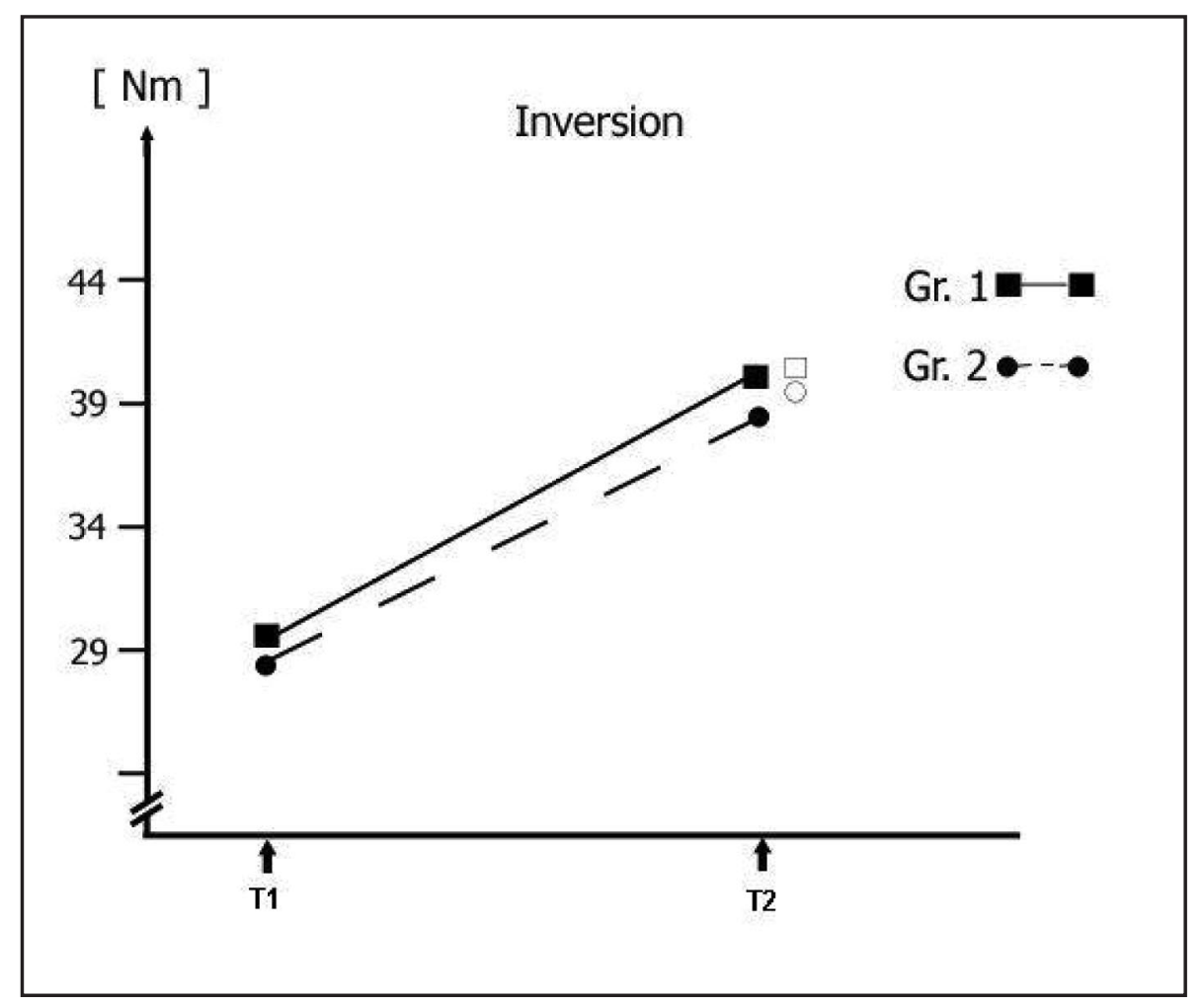

Figure 4: Maximum isokinetic torque in the inversion of the injured ankle in the comparison groups at the beginning of the investigation $\left(T_{1}\right)$ and at the end of the investigation $\left(T_{2}\right)(\square 0=$ maximum isokinetic torque on the uninjured side).

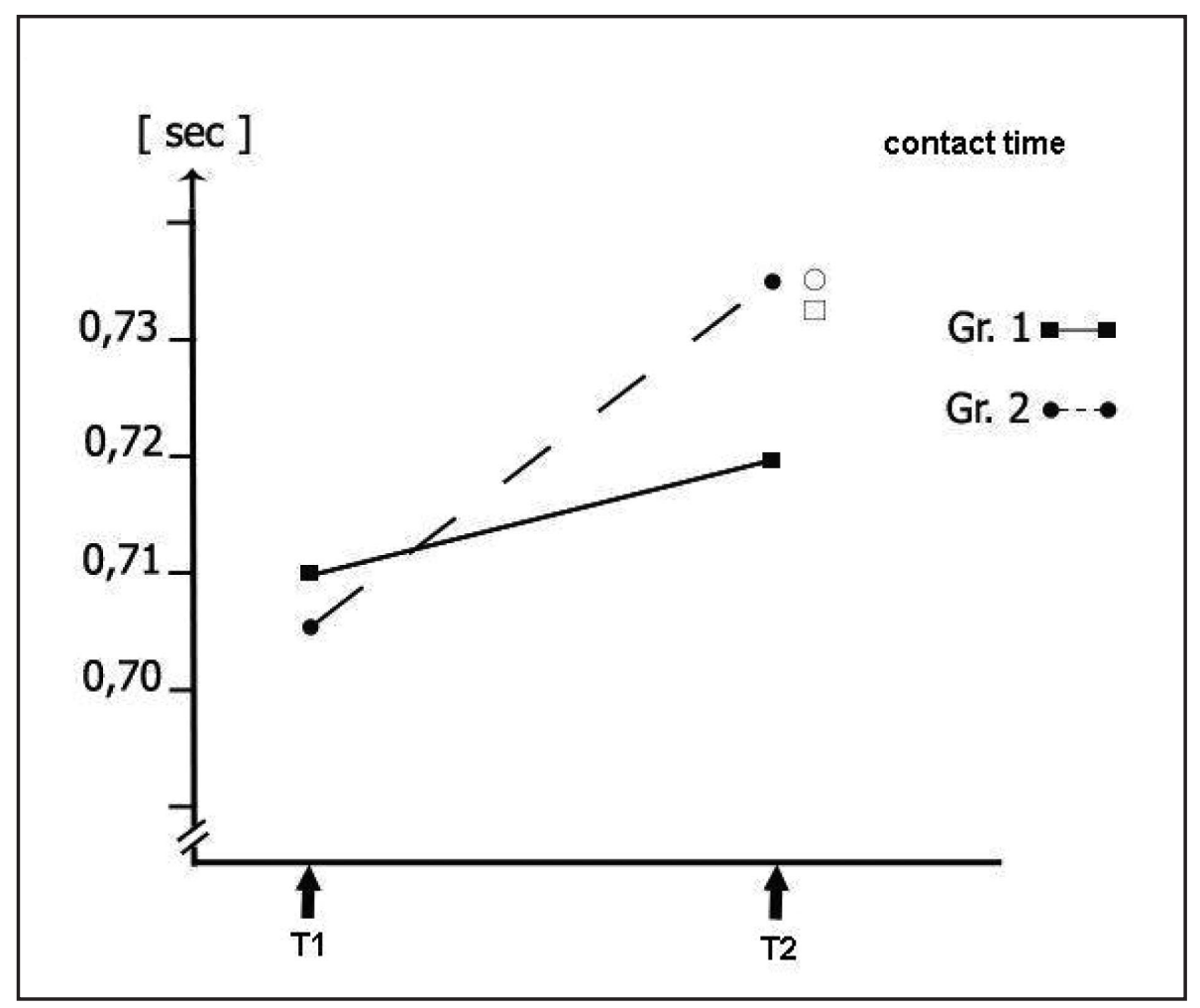

Figure 5: Contact time of the stand leg on the injured side during the gait in the comparison groups at the beginning of the investigation $\left(T_{1}\right)$ and at the end of the investigation $\left(T_{2}\right)(\square 0=$ contact tine during gait on the uninjured side in the therapy groups at the end of treatment). 
(2003). However, corresponding implementation protocols and the results of this form of training are scarcely found. Since Freeman (1965) drew attention to the term functional ankle instability, interest has been focused almost exclusively on proprioceptive training in acute ankle sprains and in chronic ankle joint instability. However, Bosien et al (1995) demonstrated a weakness of the peroneal musculature that indicates chronic ankle joint instability. Larsen and Lund (1991) could show that strength training of the peroneal musculature improves the control of the foot position as well as the neuromotor activity of these muscles. In animal experiments, Watson et al (2001) observed that passive exercises are indeed able to reduce a strength deficit. To what extent a strength deficit is simultaneously associated with a deficit in proprioception can hardly be established from the investigations up to now. However, it could be assumed from the results of the above authors that there may be a reciprocal relationship between the two phenomena. The present results indicate that a rehabilitation protocol that concentrates on proprioception training is unable to compensate for the strength deficit of the ankle musculature to a satisfactory extent and this is noticeable in dorsal extension and eversion motion (Fig. 2 and 3) that still showed pronounced deficits in the final test (T2) in group 1 compared to the healthy side. On the basis of the present investigation protocol, it is uncertain whether there were also demonstrable proprioception differences between the groups. However, the result of the significantly prolonged contact time (Fig. 5) in group 2 indicates that the weightbearing ability of the injured leg could be demonstrably optimized compared to group 1 after isokinetic strength training in group 2 . The results that were attained in these investigations were practically the same on each side. This indicates that there is a better loading function during the gait cycle which is likely to be attributable to an enhanced functional stability of the affected ankle joint. The range of motion at the second examination (Tab. 1) also indicates further postural control of the ankle joint in group 2 .
Since proprioception training was conducted during the first four posttraumatic weeks in all patients, its effects must also be considered in group 2. In the subsequent three weeks of treatment, however, the targeted isokinetic training in this group brought about a better enhancement of strength with compensation of the post-traumatic side-to-side difference and at the same time a side-to-side compensation of the shortened contact time of the injured leg in the exercise phase. These results indicate that stabilizing strength exercises are likely to be of particular significance in the ankle joint just as in instabilities of other joints. Likewise, it shows that proprioception is improved. It must be tested in further investigations to what extent the proprioception and strength at the ankle joint are interdependent.

\section{REFERENCES}

Berlemann NC, Berlemann U, Zimmermann $\mathrm{H}$ 2000 Gibt es noch eine Operationsindikation nach Sprunggelenks-Supinationstraumata? Review zur aktuellen Diagnostik und Therapie. Therapeutische Umschau 57: 748-55

Bosien WR, Stables OS, Russel SW 1995 Residual disability following acute ankle sprains. Journal of Bone and Joint Surgery 37-A: 1237-1243

Chaiwanichsin D, Lorprayoon E, Noomanoch L 2005 Star excursion balance training: Effects on ankle functional stability after ankle sprain. Journal Medical Association Thai 88: 90-93

Cochran GB 1988. Orthopädische Biomechanik. Enke, Stuttgart

Freeman MAR 1965 Treatment of ruptures of the lateral ligament of the ankle. Journal of Bone and Joint Surgery 47-B: 661-668

Kaminski TW, Buckley BD, Powers ME, Hubbard TJ, Ortiz C 2003 Effect of strength and proprioception training on eversion to inversion strength ratios in subjects with unilateral functional ankle instability. British Journal Sports Medicine 37: 410-415

Larsen E, Lund PM 1991 Peroneal muscle function in chronically unstable ankles. A prospective preoperative and postoperative electromyographic study. Clinical Orthopaedics 272:219-226
Lelie M, Zachazewski J, Browne P 1990 Reliability of isokinetic torque values for ankle invertors and evertors. Journal of Orthopaedic and Sports Physical Therapy 11: 612-616

Lohrer H, Alt W, Gollhofer A, Rappe B 2000 Verletzungen am lateralen Kapselbandapparat des Sprunggelenkes. Deutsche Zeitschrift für Sportmedizin 51: 196-203

Mattacola CG, Dwyer MK 2002 Rehabilitation of the ankle after acute sprain or chronic instability. Journal of Athletic Training 37: 413-429

Matsusaka N, Yokoyama S, Tsurasaki T, Inokuchi S, Okita M 2001 Effect of ankle disc training combined with tactile stimulation to the leg and foot on functional instability of the ankle. American Journal of Sports Medicine 29: $25-30$

Rammelt S, Grass R, Biewener A, Zwipp H 2003 Bandverletzungen am oberen Sprunggelenk. Trauma und Berufskrankheit 5: 170-178

Uh BS, Beynnon BD, Helie BV 2000 The benefit of a single-leg strength training program for the muscles around the untrained ankle. American Journal Sports Medicine 28: 415-421

Watson NC, Jejurikar SS, Kalliainen LK, Calderon MS, Urbanchek MG, Eguchi T, Kuzon WM 2001 Range of motion physiotherapy reduces the force deficit in antagonists to denervated rat muscles. The Journal of surgical research 99: 156-160

Weinstein ML 1993 An ankle protocol for second-degree ankle sprains. Military Medicine $158: 771-774$

Zöch C, Fialka-Moser V, Quittan M 2003 Rehabilitation of ligamentous ankle injuries: a review of recent studies. British Journal of Sports Medicine 37: 291-295 